\title{
Subacute Oral Toxicity Assesment of Ethanol Extract of Mariposa christia vespertilionis Leaves in Male Sprague Dawley Rats
}

\author{
Syahirah Ahmad Sayuti Nurul', Hamzah Hazilawati', Rosly Shaari Mohd ${ }^{2}$, \\ Farhan Hanif Reduan Mohd ${ }^{2}$, Mohamed Mustapha Noordin ${ }^{2}$ and Md Esa Norhaizan ${ }^{3}$ \\ 'Department of Veterinary Pathology \& Microbiology, Faculty of Veterinary Medicine, Universiti Putra Malaysia, \\ Selangor, Malaysia \\ ${ }^{2}$ Animal Livestock Research Centre, MARDI Headquarters, Selangor, Malaysia \\ ${ }^{3}$ Department of Nutrition and Dietetics, Faculty of Medicine and Health Sciences, Selangor, Malaysia
}

\begin{abstract}
The term Butterfly tea refers to decoction of Mariposa christia vespertilionis leaves which is widely consumed by cancer patients throughout Malaysia and has gained a huge popularity among Malaysians, not only cancer patients but also researchers to discover the real potential of this plant. Herein, the study is aimed at evaluating the possible toxicity in 28-day subacute oral toxicity of ethanolic extract $M$. christia vespertilionis in male Sprague Dawley rats. The 28-day subacute toxicity study was conducted to detect the no-observed adverse effect level (NOAEL). In this study, a total of 30 rats were divided into the control, 5\% DMSO (vehicle), low dose (75 mg/ $\mathrm{kg})$, medium dose $(125 \mathrm{mg} / \mathrm{kg})$ and high dose $(250 \mathrm{mg} / \mathrm{kg})$ groups. The extract was administered daily from day 1 until day 28. At the end of the study, the animals were humanely sacrificed and assessed for the effect extract of Mariposa christia vespertilionis leaves on body weight and relative organ weights and haematological, biochemical and histopathological parameters. The haematological and serum biochemical parameters for the assessment of kidney and liver injuries were carried out. Results of haematological and serum biochemistry results showed no changes in the control and treated groups. In the histopathology, evaluation of kidney tissues in all treated groups showed no significant $(p>0.05)$ lesions. In contrast to kidney, liver tissues showed significant differences $(p<0.05)$ in lesions observed in low dose $(430 \mathrm{mg})$, medium dose $(700 \mathrm{mg})$ and high dose $(1480 \mathrm{mg})$ groups with very mild, mild and mild to moderate lesion of hepatic necrosis, in the respective groups, and very mild hepatic degeneration and hepatitis were scored in all three groups.
\end{abstract}

Key words: M. christia vespertilionis, Subacute oral toxicity, NOAEL, Ethanolic extract

\section{INTRODUCTION}

Mariposa christia vespertilionis or also known as Rerama leaf is recently gained attention on its potentials to cure cancer. A decoction of the plant leaves is believed use as

Correspondence to: Hamzah Hazilawati, Department of Veterinary Pathology \& Microbiology, Faculty of Veterinary Medicine, Universiti Putra Malaysia, 43400 UPM, Serdang, Selangor Darul Ehsan, Malaysia

E-mail: hazilawati@upm.edu.my

This is an Open-Access article distributed under the terms of the Creative Commons Attribution Non-Commercial License (http:// creativecommons.org/licenses/by-nc/3.0) which permits unrestricted non-commercial use, distribution, and reproduction in any medium, provided the original work is properly cited. remedies or traditional medicine, anti-plasmodium and high cytotoxicity against Hela and MRC54 (1), inhibits neuroendocrine tumours (2) and inhibits growth of S180 tumour and H22 tumour cells (3). Although several pharmacological studies (as antiplasmodial, anti-tumour) have been carried out on this plant, there is no experimental evidence on its toxicity.

Recently, increasing interest in herbal medicines is the belief that because these medicines are natural and have been traditionally used, they are safe and harmless. Nevertheless, their natural origin is not a guarantee of safety, as concerning the risks associated with the use of herbal products have noted (4). Hence, scientific information regarding the safety of this plant for use as an alternative medicine is very important before it is further developed into a new 
medicinal herbal therapy. The toxicology evaluations of Mariposa christia vespertilionis leaves are very important in order to know the side effects especially the people who consume it as a supplement and medicine. The health promoting benefits of this herbal plant have been known and being used in many countries especially for its medicinal properties. The increasing use of this herbal plant has resulted in concerns of the safety and effectiveness. Hence, through the toxicity studies in laboratory animal, the toxicology of this plant can be determined as a safeguard to the public health and to raise public awareness on the toxicity of Mariposa christia vespertilionis leaves. The toxicology studies also provide a pre-clinical safety evaluation before it can be performed and evaluated in human.

Nowadays, this plant already been distributed and consumed in Malaysia especially by cancer patients in form of a tea bag. In this present time, there is no published research on the toxicity study of this plant. Therefore, this study aimed to detect the non-observed-adverse-effect level (NOAEL) in subacute oral toxicity of ethanol extract Mariposa christia vespertilionis leaves in male Sprague Dawley rats.

\section{MATERIALS AND METHODS}

Collection of plant material. The plant, Mariposa christia vespertilionis was obtained from MARDI research station located at Muadzam Shah, Pahang, Malaysia and had been officially gone through identification process by Dr Mohd. Firdaus Ismail from Herbarium, Institute of Bioscience, Universiti Putra Malaysia (SK3167/17).

Preparation of extraction. The plant material first washed with water thoroughly to remove dirt and soil deposits and dried under shade until complete removal of moisture content, such dried plants were powdered by mechanically and passed through sieve no 80 . Approximately $250 \mathrm{~g}$ of this dried powder of Mariposa christia vespertilionis leaves was extracted with $90 \%$ ethanol respectively by continuous hot percolation, using soxhlet apparatus. The resultant dark greenish-brown extract was then concentrated up to $100 \mathrm{~mL}$ on Rota evaporator under reduced pressure. The concentrated crude extracts were then lyophilized before being used for the study. The different doses, $75 \mathrm{mg} / \mathrm{kg}$, $125 \mathrm{mg} / \mathrm{kg}$ and $250 \mathrm{mg} / \mathrm{kg}$ of body weight to plant extracts were prepared in $5 \%$ DMSO and were used for study. The extract used in each different dose are $430 \mathrm{mg}$ for dose $75 \mathrm{mg} / \mathrm{kg}, 700 \mathrm{mg}$ for dose $125 \mathrm{mg} / \mathrm{kg}$ and $1,480 \mathrm{mg}$ for dose $250 \mathrm{mg} / \mathrm{kg}$. The yield was found to be $10 \%$ lyophilized before being used for the study.

Location of study. The rats were placed in the Animal Metabolism, Toxicology and Reproductive Centre (AMTREC), Malaysia Agricultural Research and Development Institute (MARDI) at Serdang, Selangor, Malaysia.
The experiment was designed and conducted according to ethical approved by Animal Care Unit Committee (ACUC), Malaysia Agricultural Research and Development Institute (MARDI).

\section{Subacute oral toxicity study.}

- Target animal: Male Sprague Dawley (SD) rats at 6 week-old with an average weight 170 grams were used in this study. For the toxicological or toxicokinetic properties of structurally related chemicals, male rats are likely to be more sensitive compare to female rats, thus male rats are more compatible to be selected (5). A total of six rats were systematically selected out of a population of 30 rats by systematic randomization techniques and kept in their cages for 5 days prior to dosing to allow for acclimatization to the laboratory conditions. The rats were acclimatised to the housing conditions in polycarbonate plastic cages with temperature within the range of $22 \sim 27^{\circ} \mathrm{C}$, humidity at the range $40 \sim 70 \%$ and balance of $12 \mathrm{hr}$ light/ $12 \mathrm{hr}$ dark cycle. The rats were supplied by Alchemy Supplies Sdn. Bhd, Seri Kembangan, Malaysia. Clean sterile straw bedding was provided to the animals. The animals were fed with commercially available standard pellet chow and unlimited supply of filtered drinking water.

- Methodology: Procedure for main test: Subacute toxicity study was carried out in vivo. The subacute oral toxicity study was conducted using the dose test of up and down procedure according to OECD/OCDE Test Guidelines (5). In control group, the rats received normal saline and in vehicle group the rats received $5 \%$ DMSO by oral gavages.

- Body weight: Wellness parameters of animals were observed continuously during the first $30 \mathrm{~min}$ after dosing and observed periodically (with special attention given during the first $4 \mathrm{hr}$ ) for the next $24 \mathrm{hr}$ and then daily thereafter, for 28 days. Individual weekly body weights of rats were recorded before the administration of extract on 1 st week until 4th week of the experiment. Changes in the weight of individual rat was calculated and compared with that of the control and vehicle animals. The rats were observed daily for any mortality or signs of toxicity. The rats were fasted overnight before humanely euthanized with complete exsanguination under general anaesthesia with inhalation of carbon dioxide $\left(\mathrm{CO}_{2}\right)$ gas. Organs taken out after dissection, washed thrice in normal saline and dried with folds of filter paper.

- Blood collection: Blood samples were collected once at the end of study using 23 gauge needle and $3 \mathrm{~mL}$ syringe via cardiac puncture for haematology and clinical biochemistry analyses. Liver and kidneys were collected for histopathology examination.

- Haematology analysis: Blood samples were collected into ethylenediamine tetraacetic acid (EDTA) tube. Total numbers of white blood cells (WBCs) and red blood cells 
(RBCs), and haemoglobin (HB) concentration were analysed using an automated haematology analyzer (Cell Dyn $^{\circledR} 3700$, Abbott Diagnostics, NJ, USA). Blood smear was prepared and stained with Wright stain. The differential WBC count was performed manually by counting 100 WBCs on the blood smears. Packed cell volume (PCV), icterus index, and plasma protein concentration were determined manually using standard methods. Mean corpuscular volume (MCV) and mean corpuscular haemoglobin concentration (MCHC) were calculated manually by using standard formula, $\mathrm{MCV}=(\mathrm{PCV}[\mathrm{L} / \mathrm{L}] \times 1000) / \mathrm{RBC}$ $\left(\times 10^{12} / \mathrm{L}\right)$ and $\mathrm{MCHC}=\mathrm{Hb}(\mathrm{g} / \mathrm{L}) / \mathrm{PCV}(\mathrm{L} / \mathrm{L})$.

- Serum biochemistry analysis: Blood samples were collected into plain tubes and were centrifuged (Centrifuge S417R, Eppendorf, CA, USA) for $15 \mathrm{~min}$ at 3,000 rpm to obtain the serum. The serum samples were further analysed for creatinine, urea, total bilirubin, total protein (TP), albumin (ALB), globulin, aspartate aminotransferase (AST), alanine aminotransferase (ALT), alkaline phosphatase (ALP) and creatine kinase (CK) using an automated clinical chemistry analyser (Dimension Xpand Plus, Siemens, NY, USA).

- Histopathological analysis: At the end of the experimental period, all rats were sacrificed with inhalation of $\mathrm{CO}_{2}$. Selected organs of each rat such as were collected in cold [pH 4] normal saline. The organs were cleaned from blood and weighed. The organs were then fixed in $10 \%$ neutral buffered formalin solution. The process of tissue fixation was in $48 \mathrm{hr}$ duration in clean $10 \%$ formalin solution. The organs samples were processed at the Histopathology Laboratory, Faculty of Veterinary Medicine, Universiti Putra Malaysia (UPM), Serdang, Malaysia. All of the samples were trimmed about $0.5 \mathrm{~cm}$ thickness and were placed in cassettes. Later the cassettes were placed into $10 \%$ formalin solution overnight, before they were placed and undergone series of dehydrated process for about $16 \mathrm{hr}$ in an automated processor (ASP300, Leica Biosystems, Nussloch, Germany). The samples then were embedded with paraffin to form a block by a processor machine (EG1160, Leica Biosystems). The samples were trimmed about 3 5 $\mu \mathrm{m}$ thickness using a sectioning rotary microtome RM2155, Leica Biosystems), and directly placed the tissue sectioning in $45^{\circ} \mathrm{C}$ water bath before mounting on slides. All the glass slides were labelled with a diamond
Table 1. Lesion score and percentage of affected area $\left(\mathrm{mm}^{2}\right)$

\begin{tabular}{lcc}
\hline \hline Score & Percentage lesion & Actual size lesion $\left(\mathrm{mm}^{2}\right)$ \\
\hline 0 & 0 & 0 \\
0.5 & Less than $15 \%$ & 33.75 \\
1 & $15 \sim 30 \%$ & $33.76 \sim 67.49$ \\
1.5 & $30 \sim 45 \%$ & $67.50 \sim 101.24$ \\
2 & $45 \sim 60 \%$ & $101.25 \sim 134.99$ \\
2.5 & $60 \sim 75 \%$ & $135.00 \sim 168.74$ \\
3 & More than $75 \%$ & $168.75 \sim 225.00$ \\
\hline
\end{tabular}

pen and continue mounted on a hot plate $\left(54^{\circ} \mathrm{C}\right)$ overnight. Later all slides were stained with hematoxylin and eosin (H\&E), and examined microscopically at $10 \times, 20 \times$, $40 \times, 60 \times$ and $100 \times$ magnifications. The microscopic analysis of all tissue samples were carried out as a blind study. Any histopathological changes deviant from the norm were carefully recorded.

- Lesion characteristics: Toxicological lesions such as inflammation, activated kupffer cells, hydropic degeneration, regeneration and necrosis in the liver were examined and were scored. Meanwhile in kidney tissue, toxicological lesions such as cellular cast, granular cast, protein cast, inflammation, hydropic degeneration and necrosis were examined and were scored. Lesions in both liver and kidney tissues were scored as 0 (normal), 0.5 (very mild), 1.0 (mild), 1.5 (mild to moderate), 2 (moderate), 2.5 (moderate to severe) and 3.0 (severe). The detail descriptions for lesion scoring method is presented in Table 1.

- Statistical analysis: The body weight, haematological and serum biochemical parameters, and relative organ weight results were expressed as mean \pm SEM. The data were analysed using one-way ANOVA and Tukey HSD test using statistical analysis software, IBM SPSS Statistic 22.0 (IMB Corp., NY, USA). The histopathology results were expressed as mean \pm SEM and analysed using Kruskal-Wallis test for global comparison of groups for all the parameters. Non-parametric Mann-Whitney-U test was used for comparison between two groups.

\section{RESULTS}

Body weight. The average body weight of rats in oral toxicity study of Mariposa christia vespertilionis extract

Table 2. Effect of ethanol leaf extracts of Mariposa chritia vespertilionis on the body weight (mean $\pm \mathrm{SEM}$ ) of rats at 28 days

\begin{tabular}{cccccc}
\hline \hline \multirow{2}{*}{ Week } & \multicolumn{5}{c}{ Groups } \\
\cline { 2 - 5 } & Control & Vehicle & Low dose $(430 \mathrm{mg})$ & Medium dose $(700 \mathrm{mg})$ & High dose $(1480 \mathrm{mg})$ \\
\hline 1 & $164 \pm 10.35^{\mathrm{a}}$ & $185 \pm 6.49^{\mathrm{a}}$ & $183 \pm 5.95^{\mathrm{a}}$ & $170 \pm 3.81^{\mathrm{a}}$ & $184 \pm 7.28^{\mathrm{a}}$ \\
2 & $179 \pm 8.26^{\mathrm{a}}$ & $200 \pm 8.21^{\mathrm{a}}$ & $199 \pm 6.79^{\mathrm{a}}$ & $189 \pm 3.66^{\mathrm{a}}$ & $207 \pm 6.86^{\mathrm{a}}$ \\
3 & $224 \pm 7.00^{\mathrm{a}}$ & $215 \pm 11.28^{\mathrm{a}}$ & $218 \pm 7.01^{\mathrm{a}}$ & $213 \pm 6.98^{\mathrm{a}}$ & $227 \pm 7.57^{\mathrm{a}}$ \\
4 & $233 \pm 5.52^{\mathrm{a}}$ & $220 \pm 8.59^{\mathrm{a}}$ & $222 \pm 7.65^{\mathrm{a}}$ & $217 \pm 6.39^{\mathrm{a}}$ & $228 \pm 8.39^{\mathrm{a}}$ \\
\hline
\end{tabular}

${ }^{a}$ Values in the same row with similar superscript letter were not significantly different $(p>0.05)$. 
that were taken for 2 weeks are presented in Table 2. Rats in groups $\mathrm{A}, \mathrm{B}, \mathrm{C}, \mathrm{D}$ and $\mathrm{E}$ continuously gained weight from week 1 to week 4 . There were no significant changes in body weight. All animals exhibited a normal increment in body weight without drastic difference between both control and treated groups although, after the oral administration of ethanol leaf extract of Mariposa chritia vespertilionis. The changes of the body weights were found to be statistically insignificant $(p>0.05)$. Insignificant increase in body weight of rats indicates that the administration of the extract did not affect the growth of the animals.

Mortality. No mortality was observed at 75, 125 nor $250 \mathrm{mg} / \mathrm{kg}$ body weight of of ethanolic leaf extract of Mariposa chritia vespertilionis in male Sprague Dawley rats for continuous treatment for 28-day.

Haematology analysis. Based on Table 3, there were no significant $(p>0.05)$ differences in the haemogram parameters. The PCV was not affected by all the dose lev- els studied. There were also no significant differences in haemoglobin concentration, $\mathrm{RBC}, \mathrm{MCV}, \mathrm{MCHC}$, icterus index and plasma protein at all dose levels and in all studies. In leukocytes parameters, there were also no significant $(p>0.05)$ differences in the WBC counts and differential leukocyte counts. All of values were still within the reference ranges (6).

Serum biochemistry analysis. The serum biochemical parameters grouped as renal parameters (urea, creatinine) and liver parameters (total protein, albumin, globulin, alanine aminotransferase (ALT), aspartate aminotransferase (AST) and alkaline phosphatase (ALP) are presented in Table 4. Creatinine kinase (CK) was also determined. For both liver and kidney parameters there were no significant $(p>0.05)$ differences between the control, vehicle and treated rats for all parameters measured.

Organ weight. The organ relative weight results of male Sprague Dawley rats in subacute oral toxicity study are shown in Table 5, respectively. There were no signifi-

Table 3. The haematology values (mean \pm SEM) of Sprague Dawley rats in subacute oral toxicity study of ethanolic extract of Mariposa christia vespertilionis

\begin{tabular}{|c|c|c|c|c|c|}
\hline Parameter/Group & Control & Vehicle & Low dose $(430 \mathrm{mg})$ & Medium dose $(700 \mathrm{mg})$ & High dose $(1480 \mathrm{mg})$ \\
\hline $\operatorname{RBC}\left(10^{12} / \mathrm{L}\right)$ & $8.40 \pm 0.19^{\mathrm{a}}$ & $9.53 \pm 0.17^{\mathrm{a}}$ & $9.45 \pm 0.21^{\mathrm{a}}$ & $9.45 \pm 0.21^{\mathrm{a}}$ & $9.04 \pm 0.46^{\mathrm{a}}$ \\
\hline $\mathrm{Hb}(\mathrm{g} / \mathrm{L})$ & $148.40 \pm 4.76^{\mathrm{a}}$ & $165.50 \pm 4.96^{\mathrm{a}}$ & $164.50 \pm 2.40^{\mathrm{a}}$ & $166.67 \pm 2.40^{\mathrm{a}}$ & $160.00 \pm 3.58^{\mathrm{a}}$ \\
\hline $\mathrm{PCV}(\mathrm{L} / \mathrm{L})$ & $0.40 \pm 0.02^{\mathrm{a}}$ & $0.42 \pm 0.02^{\mathrm{a}}$ & $0.43 \pm 0.01^{\mathrm{a}}$ & $0.43 \pm 0.03^{\mathrm{a}}$ & $0.41 \pm 0.01^{\mathrm{a}}$ \\
\hline MCV (fL) & $62.20 \pm 0.86^{\mathrm{a}}$ & $60.67 \pm 1.45^{\mathrm{a}}$ & $60.33 \pm 0.76^{\mathrm{a}}$ & $61.00 \pm 1.09^{\mathrm{a}}$ & $60.83 \pm 0.65^{\mathrm{a}}$ \\
\hline $\mathrm{MCHC}(\mathrm{g} / \mathrm{L})$ & $284.00 \pm 0.84^{\mathrm{a}}$ & $286.67 \pm 0.67^{\mathrm{a}}$ & $287.50 \pm 1.82^{\mathrm{a}}$ & $285.50 \pm 0.89^{\mathrm{a}}$ & $290.50 \pm 1.06^{\mathrm{a}}$ \\
\hline $\mathrm{WBC}\left(\times 10^{9} / \mathrm{L}\right)$ & $5.86 \pm 1.21^{\mathrm{a}}$ & $7.97 \pm 1.06^{\mathrm{a}}$ & $6.55 \pm 0.78^{\mathrm{a}}$ & $8.00 \pm 2.08^{\mathrm{a}}$ & $7.72 \pm 1.09^{\mathrm{a}}$ \\
\hline Neutrophils $\left(\times 10^{9} / \mathrm{L}\right)$ & $0.34 \pm 0.09^{\mathrm{a}}$ & $0.78 \pm 0.06^{\mathrm{a}}$ & $0.76 \pm 0.07^{\mathrm{a}}$ & $0.96 \pm 0.05^{\mathrm{a}}$ & $0.98 \pm 0.07^{\mathrm{a}}$ \\
\hline Lymphocytes $\left(\times 10^{9} / \mathrm{L}\right)$ & $4.62 \pm 0.07^{\mathrm{a}}$ & $6.51 \pm 0.04^{\mathrm{a}}$ & $5.30 \pm 0.06^{\mathrm{a}}$ & $6.33 \pm 0.03^{\mathrm{a}}$ & $5.96 \pm 0.08^{\mathrm{a}}$ \\
\hline Monocytes $\left(\times 10^{9} / \mathrm{L}\right)$ & $0.25 \pm 0.06^{\mathrm{a}}$ & $0.19 \pm 0.02^{\mathrm{a}}$ & $0.19 \pm 0.02^{\mathrm{a}}$ & $0.32 \pm 0.07^{\mathrm{a}}$ & $0.21 \pm 0.02^{\mathrm{a}}$ \\
\hline Eosinophils $\left(\times 10^{9} / \mathrm{L}\right)$ & $0.21 \pm 0.06^{\mathrm{a}}$ & $0.17 \pm 0.03^{\mathrm{a}}$ & $0.18 \pm 0.01^{\mathrm{a}}$ & $0.13 \pm 0.08^{\mathrm{a}}$ & $0.15 \pm 0.04^{\mathrm{a}}$ \\
\hline Basophils $\left(\times 10^{9} / \mathrm{L}\right)$ & $0.18 \pm 0.05^{\mathrm{a}}$ & $0.19 \pm 0.02^{\mathrm{a}}$ & $0.14 \pm 0.01^{\mathrm{a}}$ & $0.21 \pm 0.07^{\mathrm{a}}$ & $0.14 \pm 0.02^{\mathrm{a}}$ \\
\hline Icterus index & $2.00 \pm 0^{\mathrm{a}}$ & $2.00 \pm 0^{\mathrm{a}}$ & $2.00 \pm 0^{\mathrm{a}}$ & $2.00 \pm 0^{\mathrm{a}}$ & $2.00 \pm 0^{\mathrm{a}}$ \\
\hline Plasma protein $(\mathrm{g} / \mathrm{L})$ & $69.40 \pm 1.72^{\mathrm{a}}$ & $73.83 \pm 1.47^{\mathrm{a}}$ & $73.33 \pm 1.69^{\mathrm{a}}$ & $74.33 \pm 2.70^{\mathrm{a}}$ & $75.33 \pm 2.35^{\mathrm{a}}$ \\
\hline
\end{tabular}

${ }^{a}$ Values in the same row with similar superscript letter were not significantly different $(p>0.05)$.

Table 4. The serum biochemical parameters (mean \pm SEM) of Sprague Dawley rats in subacute oral toxicity study of ethanolic extract of Mariposa christia vespertionis

\begin{tabular}{lccccc}
\hline \hline Parameter/Group & Control & Vehicle & Low dose $(430 \mathrm{mg})$ & Medium dose $(700 \mathrm{mg})$ & High dose (1480 mg) \\
\hline Urea (mmol/L) & $5.44 \pm 0.35^{\mathrm{a}}$ & $6.33 \pm 0.36^{\mathrm{a}}$ & $6.78 \pm 0.39^{\mathrm{a}}$ & $5.63 \pm 1.15^{\mathrm{a}}$ & $6.73 \pm 0.43^{\mathrm{a}}$ \\
Creatinine $(\mu \mathrm{mol} / \mathrm{L})$ & $65.60 \pm 3.96^{\mathrm{a}}$ & $69.50 \pm 3.25^{\mathrm{a}}$ & $67.00 \pm 0.82^{\mathrm{a}}$ & $65.17 \pm 13.86^{\mathrm{a}}$ & $66.33 \pm 2.36^{\mathrm{a}}$ \\
CK (U/L) & $452.40 \pm 58.86^{\mathrm{a}}$ & $530.00 \pm 99.01^{\mathrm{a}}$ & $418.00 \pm 47.15^{\mathrm{a}}$ & $275.67 \pm 61.96^{\mathrm{a}}$ & $400.33 \pm 76.91^{\mathrm{a}}$ \\
ALT (U/L) & $48.30 \pm 1.75^{\mathrm{a}}$ & $61.25 \pm 8.86^{\mathrm{a}}$ & $52.82 \pm 2.09^{\mathrm{a}}$ & $55.90 \pm 13.69^{\mathrm{a}}$ & $53.13 \pm 3.67^{\mathrm{a}}$ \\
ALP (U/L) & $278.20 \pm 40.95^{\mathrm{a}}$ & $203.33 \pm 4.45^{\mathrm{a}}$ & $200.50 \pm 11.18^{\mathrm{a}}$ & $200.33 \pm 35.27^{\mathrm{a}}$ & $214.83 \pm 16.09^{\mathrm{a}}$ \\
AST (U/L) & $97.08 \pm 4.46^{\mathrm{a}}$ & $142.65 \pm 33.28^{\mathrm{a}}$ & $110.22 \pm 6.19^{\mathrm{a}}$ & $100.50 \pm 23.03^{\mathrm{a}}$ & $124.65 \pm 20.04^{\mathrm{a}}$ \\
Total protein (g/L) & $66.22 \pm 1.87^{\mathrm{a}}$ & $71.25 \pm 1.09^{\mathrm{a}}$ & $69.98 \pm 1.45^{\mathrm{a}}$ & $63.90 \pm 2.93^{\mathrm{a}}$ & $72.15 \pm 2.20^{\mathrm{a}}$ \\
Albumin (g/L) & $38.02 \pm 0.38^{\mathrm{a}}$ & $36.18 \pm 1.66^{\mathrm{a}}$ & $33.92 \pm 2.02^{\mathrm{a}}$ & $31.95 \pm 1.39^{\mathrm{a}}$ & $38.07 \pm 0.21^{\mathrm{a}}$ \\
Globulin $(\mathrm{g} / \mathrm{L})$ & $28.20 \pm 1.49^{\mathrm{a}}$ & $35.07 \pm 0.57^{\mathrm{a}}$ & $36.06 \pm 0.57^{\mathrm{a}}$ & $31.95 \pm 1.54^{\mathrm{a}}$ & $34.08 \pm 1.99^{\mathrm{a}}$ \\
\hline
\end{tabular}

${ }^{a}$ Values in the same row with similar superscript letter were not significantly different $(p>0.05)$. 
Table 5. The relative organs weights (mean \pm SEM) of Sprague Dawley rats in acute oral toxicity of ethanol extract of Mariposa christia vespertilionis

\begin{tabular}{lccccc}
\hline \hline Organ/Group & Control & Vehicle & Low dose $(430 \mathrm{mg})$ & Medium dose $(700 \mathrm{mg})$ & High dose $(1480 \mathrm{mg})$ \\
\hline Liver & $7.25 \pm 0.27^{\mathrm{a}}$ & $8.00 \pm 0.55^{\mathrm{a}}$ & $8.49 \pm 0.37^{\mathrm{a}}$ & $7.81 \pm 0.75^{\mathrm{a}}$ & $8.55 \pm 0.54^{\mathrm{a}}$ \\
Kidneys & $1.44 \pm 0.05^{\mathrm{a}}$ & $1.47 \pm 0.06^{\mathrm{a}}$ & $1.67 \pm 0.07^{\mathrm{a}}$ & $1.54 \pm 0.09^{\mathrm{a}}$ & $1.56 \pm 0.07^{\mathrm{a}}$ \\
Lungs & $1.67 \pm 0.15^{\mathrm{a}}$ & $1.80 \pm 0.12^{\mathrm{a}}$ & $1.90 \pm 0.14^{\mathrm{a}}$ & $1.63 \pm 0.07^{\mathrm{a}}$ & $2.09 \pm 0.15^{\mathrm{a}}$ \\
Heart & $0.77 \pm 0.04^{\mathrm{a}}$ & $0.76 \pm 0.06^{\mathrm{a}}$ & $0.89 \pm 0.02^{\mathrm{a}}$ & $0.88 \pm 0.09^{\mathrm{a}}$ & $0.92 \pm 0.06^{\mathrm{a}}$ \\
Brain & $1.13 \pm 0.05^{\mathrm{a}}$ & $1.23 \pm 0.02^{\mathrm{a}}$ & $1.33 \pm 0.05^{\mathrm{a}}$ & $1.26 \pm 0.02^{\mathrm{a}}$ & $1.25 \pm 0.02^{\mathrm{a}}$ \\
Testes & $2.32 \pm 0.05^{\mathrm{a}}$ & $2.39 \pm 0.09^{\mathrm{a}}$ & $3.04 \pm 0.21^{\mathrm{a}}$ & $3.27 \pm 0.19^{\mathrm{a}}$ & $2.91 \pm 0.21^{\mathrm{a}}$ \\
Spleen & $0.43 \pm 0.02^{\mathrm{a}}$ & $0.46 \pm 0.08^{\mathrm{a}}$ & $0.51 \pm 0.03^{\mathrm{a}}$ & $0.44 \pm 0.04^{\mathrm{a}}$ & $0.50 \pm 0.08^{\mathrm{a}}$ \\
\hline
\end{tabular}

${ }^{a}$ Values in the same row with similar superscript letter were not significantly different $(p>0.05)$.

Table 6. Lesion scores of liver and kidney of Sprague Dawley rats in subacute oral toxicity of ethanolic extract of Mariposa christia vespertilionis

\begin{tabular}{|c|c|c|c|c|c|c|c|}
\hline Organ & Mean score of lesions & Control & Vehicle & $\begin{array}{l}\text { Low dose } \\
(430 \mathrm{mg})\end{array}$ & $\begin{array}{l}\text { Medium dose } \\
\quad(700 \mathrm{mg})\end{array}$ & $\begin{array}{l}\text { High dose } \\
(1480 \mathrm{mg})\end{array}$ & $\begin{array}{l}\text { Kruskal Wallis test } \\
\text { for global comparison } \\
\text { of organ lesions among } \\
\text { groups asymptotic } \\
\text { significant }(p<0.05)\end{array}$ \\
\hline \multicolumn{8}{|c|}{ Scored and Percentage of area affected $\left(\mathrm{mm}^{2}\right)$} \\
\hline \multirow{7}{*}{ Liver } & Activated Kupffer cell & $0^{\mathrm{a}}$ & $0^{\mathrm{a}}$ & $28.35 \pm 0.20^{\mathrm{ab}}$ & $39.09 \pm 0.20^{\mathrm{b}}$ & $39.09 \pm 0.20^{\mathrm{b}}$ & 0.41 \\
\hline & $\begin{array}{l}\text { Necrosis (karyolysis and } \\
\text { eosinophilic cytoplasm) }\end{array}$ & $0^{\mathrm{a}}$ & $0^{\mathrm{a}}$ & $50.5 \pm 0.25^{\mathrm{b}}$ & $84.37 \pm 0.11^{\mathrm{c}}$ & $126.55 \pm 0.18^{d}$ & $0.01^{*}$ \\
\hline & Necrosis (Pyknotic cells) & $0^{\mathrm{a}}$ & $0^{\mathrm{a}}$ & $0^{\mathrm{a}}$ & $11.50 \pm 0.17^{\mathrm{b}}$ & $11.50 \pm 0.17^{\mathrm{b}}$ & 0.54 \\
\hline & $\begin{array}{l}\text { Degeneration } \\
\text { (vacuolated cytoplasm) }\end{array}$ & $0^{\mathrm{a}}$ & $0^{\mathrm{a}}$ & $45.16 \pm 0.21^{\mathrm{b}}$ & $28.35 \pm 0.20^{\mathrm{a}}$ & $33.75 \pm 0.18^{\mathrm{a}}$ & 0.08 \\
\hline & Regeneration & $0^{\mathrm{a}}$ & $0^{\mathrm{a}}$ & $45.16 \pm 0.21^{\mathrm{b}}$ & $45.16 \pm 0.21^{\mathrm{c}}$ & $33.75 \pm 0.22^{\mathrm{d}}$ & $0.01 *$ \\
\hline & Inflammation & $0^{\mathrm{a}}$ & $0^{\mathrm{a}}$ & $11.48 \pm 0.17^{\mathrm{b}}$ & $33.75 \pm 0.22^{\mathrm{c}}$ & $45.16 \pm 0.21^{\mathrm{d}}$ & $\mathbf{0 . 0 3} *$ \\
\hline & Mean score & 0 & 0 & $36.13 \pm 0.21$ & $40.37 \pm 0.19$ & $48.25 \pm 0.19$ & 0.13 \\
\hline \multirow{7}{*}{ Kidney } & Granular cast & $0^{\mathrm{a}}$ & $0^{\mathrm{a}}$ & $0^{\mathrm{a}}$ & $0^{\mathrm{a}}$ & $0^{\mathrm{a}}$ & 1.00 \\
\hline & Cellular cast & $0^{\mathrm{a}}$ & $0^{\mathrm{a}}$ & $0^{\mathrm{a}}$ & $0^{\mathrm{a}}$ & $0^{\mathrm{a}}$ & 1.00 \\
\hline & Protein cast & $0^{\mathrm{a}}$ & $0^{\mathrm{a}}$ & $0^{\mathrm{a}}$ & $0^{\mathrm{a}}$ & $0^{\mathrm{a}}$ & 1.00 \\
\hline & Necrosis (Pyknotic cell) & $0^{\mathrm{a}}$ & $0^{\mathrm{a}}$ & $0^{\mathrm{a}}$ & $0^{\mathrm{a}}$ & $0^{\mathrm{a}}$ & 1.00 \\
\hline & Inflammation & $0^{\mathrm{a}}$ & $0^{\mathrm{a}}$ & $0^{\mathrm{a}}$ & $0^{\mathrm{a}}$ & $0^{\mathrm{a}}$ & 1.00 \\
\hline & Hydropic degeneration & $0^{\mathrm{a}}$ & $0^{\mathrm{a}}$ & $0^{\mathrm{a}}$ & $0^{\mathrm{a}}$ & $0^{\mathrm{a}}$ & 1.00 \\
\hline & Mean score & 0 & 0 & 0 & 0 & 0 & 1.00 \\
\hline
\end{tabular}

The symbol * denotes the values were significantly different from control $(p<0.05)$. ${ }^{\mathrm{a}, \mathrm{b}, \mathrm{c}}$ Indicate values in the same row with different superscript letters were significantly different $\left(p<0.05\right.$; Mann Whitney test). $0 \mathrm{~mm}^{2}$ : none, $<33.75 \mathrm{~mm}^{2}$ : less than $15 \%,<67.49 \mathrm{~mm}$ : $15 \sim 30 \%$, < $101.24 \mathrm{~mm}^{2}: 30 \sim 45 \%,<134.99 \mathrm{~mm}^{2}: 45 \sim 60 \%,<168.74 \mathrm{~mm}^{2}: 60 \sim 75 \%$ and $>168.74 \mathrm{~mm}^{2}:$ more than $75 \%$.

cantly differences $(p>0.05)$ in organ relative weight of liver, spleen, kidneys and testes in this study.

Histopathology. The histopathological examination on liver of rats treated with the M. christia vespertilionis leaf extract revealed some abnormalities as shown in Table 6 and Fig. 1. There were significant differences $(p<0.05)$ of lesions observed for necrosis (karyolysis and eosinophilic cytoplasm), regeneration and inflammation in the treated groups $(75 \mathrm{mg} / \mathrm{kg}, 125 \mathrm{mg} / \mathrm{kg}$ and $250 \mathrm{mg} / \mathrm{kg}$ ) as tested with Kruskal-Wallis, test for the global comparison of organ toxicity among groups. By using Mann Whitney $\mathrm{U}$ test for comparison between two groups as showed as superscript in Table 6, showed medium dose and high dose were significant $(p<0.05)$ on necrosis (pyknotic) and activated Kupffer cells compare to control. Meanwhile, inflammation showed significant $(p<0.05)$ on the high dose group and low dose showed significant $(p<0.05)$ on degeneration compared to control. Scores for necrosis (karyolysis and eosinophilic cytoplasm) and regeneration showed significant $(p<0.05)$ in all treated groups compare to control group.

Due to the generalized pattern of necrosis and lesions score in liver tissues, the liver were scored base on the area affected following lesions scored. In this study, as shown in Table 7, area affected in liver tissues were sig- 
Table 7. Lesion scores based on area affected in liver of Sprague Dawley rats in subacute oral toxicity of ethanolic extract of Mariposa christia vespertilionis

\begin{tabular}{|c|c|c|c|c|c|c|c|}
\hline Organ & Area & Control & Vehicle & $\begin{array}{r}\text { Low dose } \\
(430 \mathrm{mg})\end{array}$ & $\begin{array}{l}\text { Medium dose } \\
\quad(700 \mathrm{mg})\end{array}$ & $\begin{array}{l}\text { High dose } \\
(1480 \mathrm{mg})\end{array}$ & $\begin{array}{c}\text { Kruskal Wallis test } \\
\text { for global comparison } \\
\text { of organ lesions among } \\
\text { groups asymptotic } \\
\text { significant }(p<0.05)\end{array}$ \\
\hline \multicolumn{8}{|c|}{ Scored and Percentage of area affected $\left(\mathrm{mm}^{2}\right)$} \\
\hline \multirow{4}{*}{ Liver } & Centrilobular zonal & $0.00 \pm 0.00^{\mathrm{a}}$ & $0.00 \pm 0.00^{\mathrm{a}}$ & $33.75 \pm 0.22^{b}$ & $11.48 \pm 0.17^{\mathrm{b}}$ & $0.00 \pm 0.00^{\mathrm{a}}$ & $0.05^{*}$ \\
\hline & Midzonal & $0.00 \pm 0.00^{\mathrm{a}}$ & $0.00 \pm 0.00^{\mathrm{a}}$ & $45.16 \pm 0.21^{\mathrm{b}}$ & $33.75 \pm 0.22^{\mathrm{c}}$ & $45.16 \pm 0.21^{\mathrm{b}}$ & $0.02 *$ \\
\hline & Periportal zonal & $0.00 \pm 0.00^{\mathrm{a}}$ & $0.00 \pm 0.00^{\mathrm{a}}$ & $55.94 \pm 0.28^{b}$ & $78.97 \pm 0.25^{\mathrm{c}}$ & $84.37 \pm 0.25^{\mathrm{d}}$ & $0.01 *$ \\
\hline & Mean & $0.00 \pm 0.00$ & $0.00 \pm 0.00$ & $45.16 \pm 0.24$ & $41.11 \pm 0.21$ & $43.14 \pm 0.15$ & $0.03^{*}$ \\
\hline
\end{tabular}

The symbol * denotes the values were significantly different from control $(p<0.05){ }^{a, b, c, c, d}$ Indicate values in the same row with different superscript letters were significantly different $\left(p<0.05\right.$; Mann Whitney test). $0 \mathrm{~mm}^{2}$ : none, $<33.75 \mathrm{~mm}^{2}$ : less than $15 \%,<67.49 \mathrm{~mm}^{2}$ : $15 \sim 30 \%,<101.24 \mathrm{~mm}^{2}: 30 \sim 45 \%$.
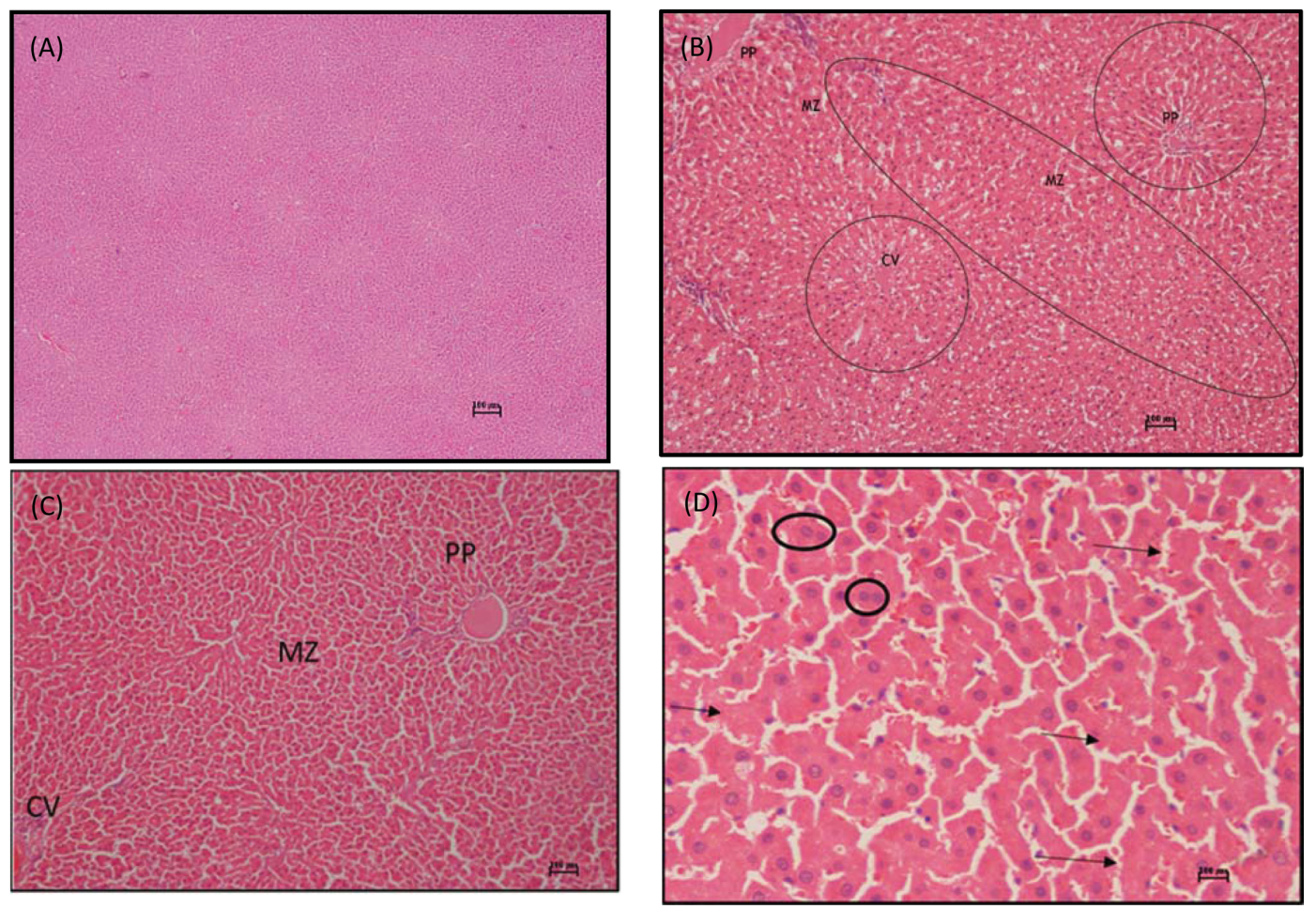

Fig. 1. (A) Photomicrograph of liver section of a control rat in subacute toxicity study of ethanolic extract of $M$. christia vespertilionis sacrified at the end of study period (H\&E stain, $\times 40)$. (B) Photomicrograph of liver section of a rat in medium dose $(125 \mathrm{mg} / \mathrm{kg})$ subacute toxicity of a ethanolic extract of $M$. christia vespertilionis sacrified at the end of study period showing necrosis at both periportal and midzonal areas (encircled) but the centrilobular area were not severed compare to both areas (H\&E stain, $\times 200)$. (C) Photomicrograph of liver section of a rat in high dose $(250 \mathrm{mg} / \mathrm{kg})$ subacute toxicity study of ethanolic extract of M. christia vespertilionis showing necrosis at all 3 area, periportal toward centrilobular area (H\&E stain, $\times 200$ ). (D) Photomicrograph of liver section of a rat in high dose $(250 \mathrm{mg} / \mathrm{kg})$ group in subacute study toxicity M. christia vespertilionis sacrified at the end of study period showed regeneration (encircled) and karyorrlysis (arrow) at periportal area (H\&E stain, $\times 400)$. C: Centrilobullar, PP: Periportal, MZ: Midzonal.

nificantly differences $(p<0.05)$ in periportal toward centrilobullar area by using Kruskal-Wallis test. However, through Mann Whitney U test, low dose and medium dose significantly $(p<0.05)$ caused periportal toward centrilobullar area were affected but in high dose only affected sig- nificantly $(p<0.05)$ at periportal toward midzonal area compare to control.

In contrast to liver lesion scoring, results of histopathological changes in the kidney tissues in this study showed no significant changes $(p>0.05)$ in all lesions (Fig. 2). 

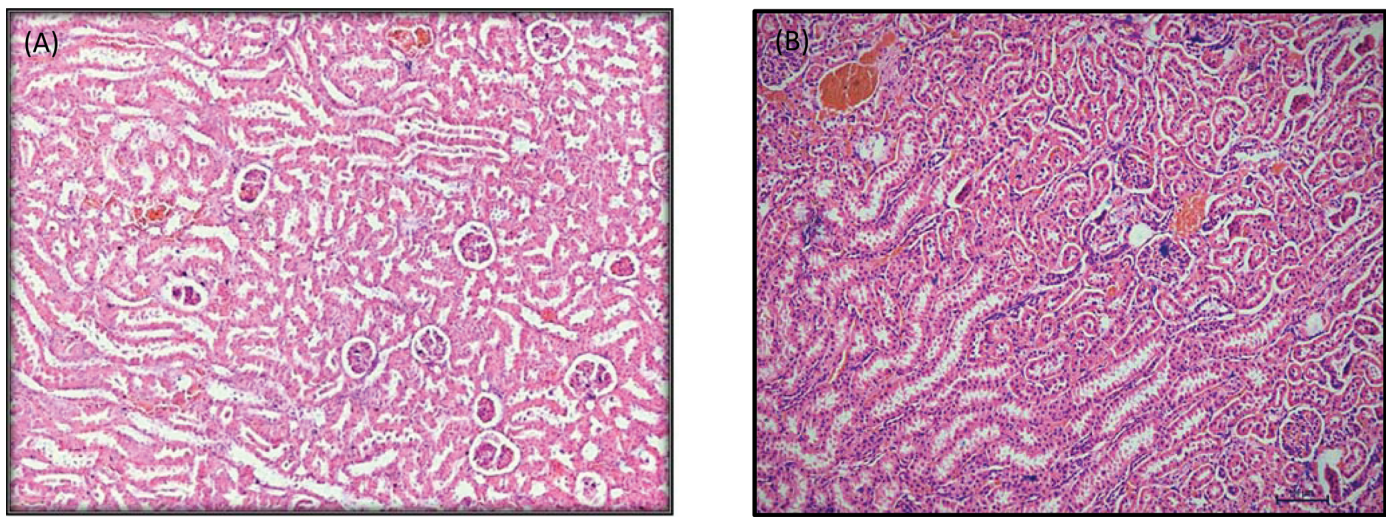

Fig. 2. (A) Photomicrograph of normal kidney section of a rat in medium dose subacute toxicity M. christia vespertilionis (H\&E stain, $\times 100$ ). (B) Photomicrograph of normal kidney section of a rat in high dose subacute study toxicity M. christia vespertilionis (H\&E stain, $\times 100)$.

\section{DISCUSSION}

In developing countries nowdays, herbal products prepared from medicinal plants are falsely considered to be safe without any compromising health effects (7). However, there is a lack of data on the toxicological profile and adverse effect of these natural compounds. Medicinal herbs are usually self-prescribed by the consumers and there is a lack of control and review in terms of dose, manner, and frequency of administration. Appropriate use of medicinal plants in dietary supplementation is very important in the maintenance of health (8). Therefore, evaluating the toxicological effects of any medicinal plant extract intended to be used in animals or humans is a crucial part of its assessment for potential toxic effects. This study focused on the herbal plant of Mariposa christia vespertilionis which has extensively been used among in Malaysian for its medicinal properties. The increased use of this plant has resulted in concerns over both the efficacy and safety of the product. It is also a useful parameter to investigating the therapeutic index of drugs and xenobiotics (9).

For the purpose of medical study experiment using plant or herb extraction, one of the keys is that all of extraction solvents and chemicals should not have toxic effects or strong interfering efforts to living cells, animals, and human beings. Extraction using the solvents of water, ethanol, ethyl acetate, and hexane, which have no toxic effects or minor interfering efforts to the living cells, animals, and human beings. Ethanol extraction product mainly contains high hydrophilic compounds including very polar neutral, basic and acidic compounds, amino acids, nucleotides, sugars, and polysaccharides. The solvent selection for isolation of any phyto-constituent depend upon its solubility in that solvent (10). Therefore this study used ethanol as solvent for purposed of extraction method.

Currently, M. christia vespertilionis already been consumed and gained attention in Malaysia especially cancer patients for their potential to cure. This plant has no clinical proven yet on the toxicity either in vivo or in vitro studies, especially on repeated-dose treatment for 28-days. Observation for mortality rate is very important in toxicity study (11). Throughout 28 days of observation period, rats in this present study showed no mortality neither signs of behavioural changes nor abnormalities were detected. This indicates that the administration of extract even on low dose, medium dose and high dose has negligible level of toxicity on the growth of the animals, same result as in study by Chung-Tak et al (12).

Body weight is one of parameters used for evaluating health status of experimental animals (13). After exposure to a few possible toxic substances, there will be changes in body weight gain and internal organ weights which would reflect toxicity animal (13). The body weight changes is a result of adverse effects of drugs and chemicals and if the body weight loss occurred is more than $10 \%$ of the initial body weight it will be considered as statistically significant animal $(13,14)$. Weekly body weights recorded on this study showed no significant changes $(p>0.05)$ between control and all treated groups. In this study, the pattern of the body weight changes in all groups showed slight reduction and increment throughout the study period. Herbal extracts can contribute suppression of animal's appetite which may lead to reduction in body weight animal (13). Decrease in body weight also associated with normal physiological adaptation responses of the body towards plant extract compounds (15). Throughout this study, regardless of the dose used $(75,125$ and $250 \mathrm{mg} / \mathrm{kg}$ of body weight), $M$. christia vespertilionis extract did not appear to affect the body weight of the rats.

Organ weight is also an important index of physiological and pathological status. The relative organ weight is fundamental to evaluate whether the organs were injured or not. Organ relative weight is used to evaluate organ injury (16) which either atrophy or hypertrophy or swell- 
ing $(17,18)$. The heart, lungs, liver, kidney and spleen are the primary organs affected by metabolic reaction caused by toxicant (18). In the present study, liver and kidneys were evaluated microscopically. Liver and kidneys are two vital organs function for healthy survival of body acts as metabolic detoxification. The organ relative ratio to body weight in this study showed no significant differences $(p>0.05)$ compare control to treated groups, implying that the extract did not appear to affect the rats' organs. It can be suggested that $M$. christia vespertilionis extract is virtually nontoxic.

Haematology results in this study showed there were no significant differences between control and treated groups. This study showed no significant alterations in the haematological values which indicate that the extract up to concentration of $250 \mathrm{mg} / \mathrm{kg}$ orally daily for 28-day did not affect the haematopoietic system. Levels for all parameters were within the normal range as reported by Petterino and Argentini-Storino (6). Analysis of blood parameters is important in the evaluation of risks associated with test compounds under investigation as the changes in the haematological system have a greater indicative value for human toxicity, when the data are converted from animal studies (19). Haematopoietic system is one of the most sensitive target organ of toxic compounds (20). Evaluation of the haematological parameters can be used to determine the extent of deleterious effects of foreign compounds including plant extracts, on the blood constituents of an animal $(21,22)$. As an example, significant decrease in the $\mathrm{WBC}, \mathrm{RBC}, \mathrm{Hb}$ and $\mathrm{PCV}$ value in a study which was induced by ethanolic extract of Dennettia tripetala fruit, result as present of compound alkaloid, saponins, flavonoids, tannins and phenols in the extract may have suppressed the growth and differentiation factors in the bone marrow (23). Another probable reason for the observed decrease in RBC counts may be due to haemolysis mediated via the phytochemical components of the extract, or may be the extract caused failure of erythropoietin production, could lead to anaemia.

The application of blood serum or plasma enzymes as marker to measure organ damage, cell damage, enzyme induction, activation or inhibition of enzymes is becoming very common in toxicology studies. A variety of blood measurements could be used to evaluate the severity of tissue damage, possible target organs and to measure impairs organ functions. With the combination of these tests, a broad range of evaluation information could give an indication on their physiological and metabolic functions. Kidney and liver are two major organs that play role in metabolic detoxification. An estimation of some biochemical parameters such as activities of enzymes in tissues and blood plays major roles in disease and liver toxicity (24). There were no significant differences $(p>0.05)$ in the levels of AST, ALT, ALP, CK, bilirubin and total protein between the control and treated groups. Following liver parameters, both kidney parameters; urea and creatinine also showed no significant differences $(p>0.05)$, suggest that subacute administration of $M$. christia vespertilionis leaf extract did not cause any damage to the liver and kidney.

Apart from the blood analysis, histopathological analysis provides supportive evidence for biochemical and haematological assessments (25). The histopathologic examination is the gold standard for evaluating treatment related pathological changes in tissues and organs (26). Histolpathological assessment on kidney lesion scores in this study showed no significant $(p>0.05)$ changes detected on the control and all treated groups. However, histopathological assessment of $M$. christia vespertillionis extract in treated samples of liver in this present study showed necrosis (karyolysis, eosinophilic cytoplasm and pyknotic cells), degeneration and inflammation. The possibility that these changes may reflect a cellular adaptation beneficial of extract to the host tissue. Degeneration of liver cells following low doses of this extract was due to excess accumulation of glycogen, predominantly of the monoparticulate form (27). This tolerance to toxic damage seemed to be associated with excess accumulation of intracellular glycogen. This can be observed on animal and human livers that many of the vacuolated hepatocytes seen in liver injury (27). Structural changes scores for necrosis with atrophy of hepatocytes will be result of impairment of venous outflow $(28,29)$. This impairment can occur at the level of the small hepatic veins (veno-occlusive disease), large hepatic veins (Budd-Chiari syndrome), or the heart (right-sided heart failure, tricuspid valve disease, constrictive pericarditis) (30-32). However, these changes were most pronounced in generalised area, but most prominent at periportal towards midzonal in all 3 doses and were often accompanied by eosinophilic cytoplasm, karyolysis, inflammation and activated Kupffer cells. Activated Kupffer cells and inflammatory cells presence due to the reaction of the host toward toxicant as respond on liver injury (33-35). Eosinophilic cytoplasm of hepatocytes were seen in degenerated hepatocytes especially at the junction of the necrotic areas and normal parenchyma due to xenobiotic-induced effect (36-39).

Based on the results, all doses showed significant on the regeneration score when compared to control. Regeneration is due to reaction of the hepatocytes toward toxicant $(38,40)$. In this study, scores for regeneration most outstanding at the periportal towards midzonal area as result to replace the hepatocytes that affected at this area. As an example, Phyllanthus amarus Linn, induces regeneration or mitotic division of hepatocyte after acute liver injury (38). Besides, possible bioactive compounds from the same family of Papilonaceae are alkaloid, phenols and phytosterol or stigmasterol. These all bioactive compounds can be lead to acute hepatocellular injury. Alkaloids are 
plant metabolites that have a nitrogen-containing chemical ring structure, alkali-like chemical reactivity, and pharmacologic activity (1). React in cellular protein and DNA leading to hepatotoxicity. As an example, pyrrolizidine alkaloid (PA) one of the bioactive compounds belong to this herb's family, reported have minimal toxicity in their original form, metabolized in the liver and can become toxic metabolites, depending on the PA and on the particular condition of the liver enzymes $(39,41,42)$.

The liver is prone to xenobiotic-induced injury because of its central role in xenobiotic metabolism, portal location within the circulation and its anatomic and physiologic structure $(40,43)$. Most drugs are not intrinsically toxic to the liver but can cause injury secondary to the production of an hepato-toxic drug metabolite, a process known as bioactivation $(43,44)$. Based on the results in this study, can be seen this M. christia vespertilionis showed action as renoprotective, because no significant lesion score in this study. This present study followed acetaminophen (paracetamol), associated liver damage is more recognized than kidney damage (45). The mechanism of liver injury is related to the fact that small amounts of acetaminophen are converted to a toxic metabolite, which later binds with liver proteins to cause cellular injury. The amount of toxic metabolite produced and the ability of the liver to remove this metabolite before it binds to liver protein influence the extent of liver injury. This overdose and long term (chronic) of acetaminophen will result on nephrotoxicity (46).

Plants can contain pharmacologically useful and active compounds, but unsurprisingly, they can also contain toxic substances. As an example, phenol or catechin in green tea consumption cause the hepatotoxicity. Accumulated metabolites apparently have the property of capturing free radicals, a process which may lead to liver toxicity, damage to the hepatocyte's mitochondria and difficulty in metabolizing (47). Phytosterol or stigma sterol will promote liver injury and activated of Kupffer cells (47). Based on the results on kidney tissues, the observations seem to suggest that certain medicinal plants might have renal protective ability to prevent kidney dysfunction by accelerating regeneration (47). There are two possible effects of various herbs on kidneys, harmful or beneficial. Harmful effects include, polyuria, causing dehydration, acute renal failure, chronic renal insufficiency and stone formation. Possible beneficial effects include, dieresis, protection of the kidney from nephrotoxic agents, prevention or amelioration of renal lithiasis and amelioration of kidney failure (48). Some herbs such as $R$. decursiva extracts did not show any signifcant toxicological changes as observed by histopathological examination in the kidney and liver tissues even though for subacute (28-day) and subchronic (90-day) toxicity study (48). This most probably because of the bioactive compounds in the extract were not cause toxic to the rats. There are various mechanisms by which renoprotection may be achieved and anti-oxidative properties seem to be very important. Kidney damage is usually associated with an increase in oxidative stress and the oxidative stress induced kidney injury is reduced by antioxidants. Increased activities and levels of antioxidants including phenolic compounds and carotenoids reduce kidney injury (49).

In conclusion, no mortality occurred in this subacute oral dose toxicity study. The NOAEL of this extract to liver in this 28-day subacute toxicity study was low than $75 \mathrm{mg} / \mathrm{kg}$ (430 mg used extract) while for kidney NOAEL will be greater than $250 \mathrm{mg} / \mathrm{kg}$ (1,480 mg used extract). These studies aimed at acquiring safety data for the application of a natural substance based medicine, including information about the NOAEL and target organs. Results demonstrate the safety of subacute oral administration of ethanolic leaf extract of $M$. christia vespertilionis in rats and indicate that future long-term toxicity (subchronic 90day) testing of $M$. christia vespertilionis is warranted.

\section{ACKNOWLEDGMENTS}

The authors would like to thanks Universiti Putra Malaysia (UPM), and Malaysian Agriculture and Development Institute (MARDI), Malaysia for providing facilities and fund for this project.

\section{CONFLICT OF INTEREST}

The authors declare that the research was conducted in the absence of any commercial or financial relationships that could be construed as a potential conflict of interest.

Received July 23, 2017; Revised December 2, 2017; Accepted January 9, 2018

\section{REFERENCES}

1. Nguyen-Pouplin, J., Tran, H., Phan, T.A., Dolecek, C., Farrar, J., Tran, T.H., Caron, P., Bodo, B. and Grellier, P. (2007) Antimalarial and cytotoxic activities of ethnopharmacologically selected medicinal plants from South Vietnam. J. Ethnopharmacol., 109, 417-427.

2. Hofer, D., Schwach, G., Ghaffari Tabrizi-Wizsy, N., Sadjak, A., Sturm, S., Stuppner, H. and Pfragner, R. (2013) Christia vespertilionis plant extracts as novel antiproliferative agent against human neuroendocrine tumor cells. Oncol. Rep., 29, 2219-2226.

3. Wu, X.-Y., Tang, A.-C. and Lu, Q.-Y. (2012) Study on antitumor effect of the extract from Christia vespertilionis in vivo. Chinese Journal of Experimental Traditional Medical Formulae, 2012-08.

4. Whiting, P.W., Clouston, A. and Kerlin, P. (2002) Black cohosh and other herbal remedies associated with acute hepatitis. Med. J. Aust., 177, 440-443. 
5. Organization for Economic Cooperation and Development (2001) Guidance document for testing chemicals. Acute oral toxicity study-fixed dose.

6. Petterino, C. and Argentino-Storino, A. (2006) Clinical chemistry and haematology historical data in control Sprague Dawley rats from pre-clinical toxicity studies. Exp. Toxicol. Pathol., 57, 213-219.

7. Harizal, S.N., Mansor, S.M., Hasnan, J., Tharakan, J.K.J. and Abdullah, J. (2010) Acute toxicity study of the standardized methanolic extract of Mitragyna speciosa Korth in rodent. J. Ethnopharmacol., 131, 404-409.

8. Wang, D., Zhong, K.Y.Z., Luo, X., Xiao, R., Hou, Y., Bao, W., Yang, W., Yan, H., Yao, P. and Liu, L. (2011) Acute and subchronic oral toxicities of Pu-erh black tea extract in Sprague-Dawley rats. J. Ethnopharmacol., 134, 156-164.

9. Rang, H.P., Dale, M. and Ritter, R. (2001) Pharmacology (volume 13, 4th edition), Churchill Livingstone, New York.

10. Handa, S.S., Khanuja, S.P.S., Longo, G. and Rakesh, D.D. (2008) Extraction Technologies for Medicinal and Aromatic Plants (1st edition, no. 66), United Nations Industrial Development Organization and the International Centre for Science and High Technology, Italy.

11. Teo, S.D., Stirling, S., Thomas, A., Kiorpers, A. and Vikram, K. (2002) A 90-day oral gavage toxicity study of D-methylphenidate and D, L methylphedinate in Sprague Dawley rats. Toxicology, 179, 183-196.

12. Han, C.T., Kim, M.J., Moon, S.H., Jeon, Y.R., Hwang, J.S., Nam, C., Park, C.W., Lee, S.H., Na, J.B., Park, C.S., Park, H.W., Lee, J.M., Jang, H.S., Park, S.H., Han, K.G., Choi, Y.W., Lee, H.Y. and Kang, J.K. (2015) Acute and 28-day subacute toxicity studies of hexane extracts of the roots of lithospermumerythrorhizon in sprague-dawley rats. Toxicol. Res., 31, 403-414.

13. Raza, M., Al-Shabanah, O.A., El-Hadiyah, T.M. and AlMajed, A.A. (2002) Effect of prolonged vigabatrin treatment on hematological and biochemical parameters in plasma, liver and kidney of Swiss albino mice. Sci. Pharm., 70, 135145.

14. Carol, S.A. (1995) Acute, subchronic and chronic toxicology in CSR Handbook of Toxicology (Michael, J.D. and Mannfred, A.H. Eds.). CSR Press Inc., Boca Raton, FL, USA, pp. 51-104.

15. Rosidah, Y., Sdikun, A., Ahmad, A., Akowuah, G.A. and Asmawi, M.Z. (2009) Toxicology evaluation of standardized methanol extract of Gynuraprocumbens. J. Ethnopharmacol., 123, 244-249.

16. Amresh, G.R., Singh, P.N. and Rao, C.V. (2008) Toxicological screening of traditional medicine Laghupatha (Cissampelos pareira) in experimental animals. J. Ethnopharmacol., 116, 454-460.

17. Mohamed, E.A.S., Lim, C.P., Ebrika, O.S., Asmawi, M.Z., Sadikun, A. and Yam, M.F. (2011) Toxicity evaluation of a standardised 50\% ethanol extract of Orthosiphonstamineus. J. Ethnopharmacol., 133, 358-363.

18. Dybing, E., Doe, J., Groten, J., Kleiner, J. and O'Brien, J. (2002) Hazard charecterization of chemicals in food and diet: dose response, mechanism and extrapolation issues. Food Chem. Toxicol., 42:237-282.

19. Olson, H., Betton, G., Robinson, D., Thomas, K., Monro, A.,
Kolaja, G., Lilly, P., Sanders, J., Sipes, G., Bracken, W., Dorato, M., Van Deun, K., Smith, P., Berger, B. and Heller, A. (2000) Concordance of the toxicity of pharmaceuticals in humans and in animals. Regul. Toxicol. Pharmacol., 32, 5667.

20. Adeneye, A.A., Ajagbonna, O.P., Adeleke, T.I. and Bello, S.O. (2006) Preliminary toxicity and phytochemical studies of the stem bark aqueous extract of Musangacecropioides in rats. J. Ethnopharmacol., 105, 374-379.

21. Diallo, A., Gbeassor, M., Vonor, A., Eklu-Gadegbeku, K. and Aklikokou, A. (2008) Effect of Tectonagrandis on phenylhydrazine-induced anaemia in rats. Fitoterapia, 79, 332-336.

22. Ashafa, A.O.T., Orekoya, L.O. and Yakubu, M.T. (2012) Toxicity profile ethanolic extract of Azadirachtaindica stem bark in male Winstar rats. Asian Pac. J. Trop. Biomed., 2, 811-817.

23. Daniel, E.I. and Clement, O.N.K.U. (2008) Effect of ethanolic extract of Dennettiatripetala fruit on haematological parameters in albino wistar rats. Niger. J. Physiol. Sci., 23, 13-17.

24. Malomo, S.O. (2000) Toxicological implications of certriaxone administration in rats. Nigerian Journal of Biochemistry and Molecular Biology, 15, 33-38.

25. Organization for Economic Cooperation and Development (2001) Guideline for testing of chemicals, No. 420.

26. Larrey, D. (2002) Epidemiology and individual susceptibility to adverse drug reactions affecting the liver. Semin. Liver Dis., 22, 145-155.

27. Friedel, R., Bode, R. and Trautschold, I. (1976) Distribution of intravenously injected enzymes of heterologous, homologous and autologous origin. Distribution and transport of cell enzymes within the extracellular space. J. Clin. Chem. Clin. Biochem., 14, 129-136.

28. Nayak, N.C., Sathar, S.A., Mughal, S., Duttagupta, S., Mathur, M. and Chopra, P. (1996) The nature and significance of liver cell vacuolation following hepatocellular injury-an analysis based on observations on rats rendered tolerant to hepatotoxic damage. Virchows Arch., 428, 353-365.

29. Tanaka, M. and Wanless, I.R. (1998) Pathology of the liver in Budd-Chiari syndrome: portal vein thrombosis and the histogenesis of veno-centric cirrhosis, veno-portal cirrhosis, and large regenerative nodules. Hepatology, 27, 488-496.

30. Iwai, M., Kitagawa, Y., Nakajima, T., Takeda, M., Okanoue, T., Nakajima, T., Ogasawara, T., Suyama, Y., Miyoshi, M. and Kashima, K. (1998) Clinical features, image analysis, and laparoscopic and histological liver findings in BuddChiarI syndrome. Hepatogastroenterology, 45, 2359-2368.

31. Naschitz, J.E., Slobodin, R.J. and Yeshurun, D. (2000) Heart diseases affecting the liver and liver diseases affecting the heart. Am. Heart J., 140, 111-120.

32. Radosavljevi, T., Nikoli, I., Todorovi, V., Sikic, B. and Nikoli, J.A. (2001) Effects of portacaval shunt on theenteroinsular axis in rats. Acta Vet. Scand., 51, 275-282.

33. Bruguera, M., Aranguibel, F., Ros, E. and Rodes, J. (1978) Incidence and clinical significance of sinusoidal dilatation in liver biopsies. Gastroenterology, 75, 474-478.

34. Poulsen, H., Winkler, K. and Christoffersen, P. (1970) The significance of centrilobular sinusoidal changes in liver biopsies. Scand. J. Gastroenterol. Suppl., 7, 103-109. 
35. Djaldetti, M. and Feller, N. (1978) Crystalline cytoplasmic inclusions in the liver cells of two mongrel dogs. Res. Exp. Med. (Berl.), 173, 279-283.

36. Koller, L.D. (1973) A note on eosinophilic cytoplasmic bodies in the liver of a rabbit. Vet. Pathol., 10, 295-298.

37. Ladds, P.W. and Strafuss, A.C. (1971) Eosinophilic cytoplasmic bodies in a bovine liver. Cornell Vet., 61, 486-489.

38. Chattopadhyay, P., Agrawal, S.S. and Garg, A. (2006) Liver regenerative effect of Phyllanthus amarus Linn. against alcohol induced liver cell injury in partially hepatectomised albino rats. Int. J. Pharm., 2, 426-430.

39. Chen, Z. and Huo, J.R. (2010) Hepatic veno-occlusive disease associated with toxicity of pyrrolizidine alkaloids in herbal preparations. Neth. J. Med., 68, 252-260.

40. Cullen, J.M. (2005) Mechanistic classification of liver injury. Toxicol. Pathol., 33, 6-8.

41. Greaves, P., Edwards, R., Cohen, G.M. and MacFarlane, M. (2001) "Have you seen this?" Diffuse hepatic apoptosis. Toxicol. Pathol., 29, 398-400.

42. Bernhardt, R. (1995) Cytochrome P450: structure, function, and generation of reactive oxygen species. Rev. Physiol. Biochem. Pharmacol., 127, 137-221.

43. Lu, H., Meng, X., Li, C., Sang, S., Paten, C., Sheng, S., Hong, J., Bai, N., Winnik, B., Ho, C.T. and Yang, C.S. (2003) Glucuronides of tea catechins: enzymology of bio- synthesis and biological activities. Drug Metab. Dispos., 31, 452-461.

44. Vessey, D.A. (1996) Metabolism of xenobiotics by the human liver in Hepatology: a Textbook of Liver Disease (3rd edition), (Zakin, D. and Boyer, T.D. Eds.). WB Saunders, Philadelphia, pp. 257-305.

45. Mitchell, J.R., Jollow, D.J., Potter, W.Z., Davis, D.C., Gillette, J.R. and Brodie, B.B. (1973) Acetaminophen-induced hepatic necrosis. I. Role of drug metabolism. J. Pharmacol. Exp. Ther., 187, 185-194.

46. Mazer, M. and Perrone, J. (2008) Acetaminophen-induced nephrotoxicity: pathophysiology, clinical manifestations, and management. J. Med. Toxicol., 4, 2-6.

47. Miyaoka, Y. and Miyajima, A. (2013) To divide or not to divide: revisiting liver regeneration. Cell Div., 8, 8 .

48. Siti, S.A., Norhaizan, M.E. and Hazilawati, H. (2014) Histopathologic changes in liver and kidney tissues from male sprague dawley rats treated with Rhaphidophora decursiva (Roxb.) schott extract. J. Cytol. Histol., S4, 001.

49. Ghule, A.E., Jadhav, S.S. and Bodhankar, S.L. (2011) Renoprotective effect of Linumusitatissimum seeds through haemodynamic changes and conservation of antioxidant enzymes in renal ischaemia-reperfusion injury in rats. Arab. J. Urol., 9, 215-221. 